\title{
Introduction to HICSS-55 Business Intelligence, Business Analytics and Big Data: Innovation, Deployment and Management Minitrack
}

\author{
Olivera Marjanovic \\ University of Technology Sydney \\ Australia \\ olivera.marjanovic@uts.edu.au
}

\author{
Barbara Dinter \\ Chemnitz University of Technology \\ Germany \\ barbara.dinter@wirtschaft.tu- \\ chemnitz.de
}

\author{
Thilini Ariyachandra \\ Xavier University \\ USA \\ ariyachandrat@xavier.edu
}

New developments and the evolution of data and technologies in the business intelligence (BI)/ business analytics (BA) and big data space have given rise to new opportunities and challenges for individuals, organizations and society. As a result of rapid digital transformation, organizations are now faced with various challenges, covering the whole life cycle of $\mathrm{BI} / \mathrm{BA} / \mathrm{big}$ data applications and projects. Moreover, in the age of the pandemic, analytics driven data democratization and analytics-enabled digital resilience are shifting traditional data governance practices and the data driven culture in organizations. At the same time, companies are experiencing an unprecedented shortage in skills and analytical capabilities, which in turn limit their potential.

In addition, the growth in this space has led to societal implications, both positive and negative, that are felt strongly by more organizations, communities and societies in yet-to-be understood ways. The ongoing evolution of this field has created a state of flux, with new opportunities and challenges constantly emerging.

To close the gap between potential and real benefits of $\mathrm{BI} / \mathrm{BA} / \mathrm{big}$ data, more research on their deployment is needed in terms of artifacts such as methods, reference architectures, etc. as well as theoretical and conceptual underpinnings. Finally, operations and continuous enhancement of $\mathrm{BI} / \mathrm{BA} / \mathrm{big}$ data applications encompass management tasks that consider strategic, economic, legal, societal, ethical, and more recently, human rights issues.

This year's minitrack includes the following nine research papers:

The opening paper by Marjanovic, Ariyachandra and Dinter invites the readers to look ahead and consider business intelligence \& analytics (BI\&A) in the post-pandemic new normal. The authors identify twelve key post-pandemic BI\&A trends from industry practice and six major research themes. The paper also offers an initial set of research questions for future BI\&A research in the postpandemic new normal.

The second paper by Baars, Weber and Tank focuses on data sharing among Small and Medium Enterprises (SMEs). The authors claim that limited success in this domain could be attributed to a lack of institutionalized governance structures. Based on their research insights from a qualitative study, the authors identify the key stakeholders and conceptualize their roles.

In the third paper, Elsman and Borgman investigate how and why organizational Big Data Analytics (BDA) initiatives evolve over time in established firms. Based on the research findings from seven case studies, the authors identify four constructs: analytics structure, leadership role, culture and employees' skills, which influence organizational diffusion of BDA.

Based on survey data gathered from IT and business managers from 112 Belgian and Dutch firms, De Rijck found a direct relationship between a firm's Big Data Analytics Capability (BDAC) and its competitive advantage. A partial mediation of this relationship was found to be in the performance of the firm's operation management processes.

Focusing on the gap between Big Data Analytics (BDA) Challenges and BDA Capability, Hirschlein, Meckenstock and Dremel used a systematic literature review to identify five dimensions of BDA implementation challenges. By observing these challenges through the lens of a BDA capability, the authors articulated key propositions about possible ways to address them.

Abhari, Davis, Ness, Pagador, Parsons and Brodskiy focus on data literacy of non-expert endusers. Using Transformative Learning Theory (TLT) and Experiential Learning Theory (ELT), the authors propose an innovative data education framework 
aimed at increasing data literacy across organizations. Informed by the interviews conducted with individuals from the U.S. Department of Defense, the proposed model includes four strategies for data literacy training: experiential data training, critical incident reflection, rational open discourse, and autonomous experimentation.

Starting from the relationship between data value and quality, which they observed in related literature, Stein and Maass conducted semi-structured expert interviews to gain better insights into this relationship. Based on their findings, the authors proposed eleven requirements for data valuation methods and seven value-driving quality criteria. The paper also offers a number of future challenges related to data valuation.

Linking reference process models and data-driven design Meyer, Wiederkehr, Panzner, Koldewey and Dumitrescu propose a reference process model for data-driven product planning. To demonstrate how it could be used in practice, the authors apply the proposed model to a manufacturing company.
The final paper by Volk, Staegemann, Islam and Turowski investigates big data system architecture deployments. To overcome the observed challenges of high costs, limited modifiability and a risk of vendor lock-ins, the authors propose modern container and task automation technologies that could be used to wrap complex big data technologies into reusable and portable resources. The authors also propose a framework for deployment of big data architecture in organizations with limited resources.

We trust that you will find this year's selection of very diverse papers thought-provoking and relevant for your own research, education and/or professional practice. We look forward to receiving your research in the future. 